\title{
A proposed approach to correlate antibiotic and biocide resistance in staphylococcal isolates based on Minimum Inhibitoy Concentration (MIC) data analyses \\ Fathy M. E. Serry*a, Eman M. Elmasry ${ }^{\mathrm{a}}$, Ahmed M. Elwan ${ }^{\mathrm{a}}$ \\ Department of Microbiology and Immunology, Faculty of Pharmacy, Zagazig University, Egypt \\ * Correspondence author, Dr. Fathy M. E. Serry, Professor of Microbiology, Faculty of Pharmacy, Zagazig University, Zagazig, Egypt. E mail: fathyserry@ hotmail.com
}

\begin{abstract}
The study aimed to compare the sensitivity of Staphylococcus isolates from hospital and non hospital sources to some commonly used antibiotics and biocides, and to investigate whether resistance to either types of antimicrobials are correlated. The minimum inhibitory concentrations (MICs) of the antibiotics and biocides were determined for clinical (47), hospital environment (64) and non hospital (33) isolates using agar dilution method. Arithmetic progression in biocides concentration was used instead of the geometric progression for antibiotics. Pearson's correlation coefficients (r) between MIC-MIC, Log MIC-Log MIC, and MIC-Log MIC for individual biocides and antibiotics, respectively, were compared. Also, the frequencies of antibiotic resistance among biocide-susceptible and biocide-resistant isolates were calculated. The antibiotics included: ampicillin, penicillin G, oxacillin, cefepime, streptomycin, tetracycline, gentamicin, azithromycin, ciprofloxacin, vancomycin and chloramphenicol, while biocides included chlorocresol, benzalkonium chloride, cetrimide, chlorhexidine, phenyl mercuric nitrate, povidone-iodine and ethidium bromide. Hospital isolates showed higher resistance rates to antibiotics and biocides and more predominance of methicillin resistance compared to non-hospital isolates. The most likely correlation between antibiotic and biocide resistance was best expressed by comparing log MIC of antibiotic with MIC of biocide. For hospital isolates, positive correlations were found between increased resistance to most of tested antibiotics (except for vancomycin and occasionally ciprofloxacin and tetracycline) and reduced susceptibility to biocides, except for chlorhexidine and povidone-iodine. For non hospital isolates, only resistance to benzalkonium chloride and cetrimide correlated with resistance to most of the tested antibiotics. Except for chlorhexidine and povidoneIodine higher resistance rates to antibiotics were found among biocide resistant isolates.
\end{abstract}

Keywords: co-resistance; antibiotics; biocides; staphylococcus, MRSA; hospital; non-hospital

\section{INTRODUCTION}

Microbial resistance to antibiotics is an increasing problem. Some evidences suggest that exposure to biocides (antiseptics and disinfectants) used in various setting may contribute to the increased occurrence of antibiotic resistance (Russell et al., 1998 \&1999; Fraise 2002; Walsh et al., 2003; Sheldon et al.,2005; Weber and Rutala, 2006; Carson et al., 2008).

Antibiotic and biocide antibacterial actions show many similarities despite some differences in terms of target, killing behaviour, clinical aspects (Poole 2007) and definition of resistance (McDonnell and Russell, 1999; Russell, 2003). Some mechanisms of resistance, like efflux pumps, are common to both biocides and antibiotics and have been shown to act on a range of chemically dissimilar compounds (Levy, 2002; Maillard, 2007; Poole, 2007).

Although the role of biocide use on development of antibiotic resistance is believed to be over emphasized (Jones, 1999), research on the impact of biocide use on spread of antimicrobial resistance is urged (the Scientific Committee on Emerging and Newly Identified Health Risks, SCENIHR, 
2009). However, there are no standardized testing protocols that measure both biocide and antibiotic resistance in bacteria (SCENIHR, 2009). Although many reports of bacterial resistance to biocides are based on MIC data, using MIC to measure bacterial resistance is arguable (Russell and McDonnell 2000, Russell et al., 1998). Unlike antibiotic, where resistance is manifested by very sharp change in MIC due to lack of or reduced susceptibility to the specific target, the biocide can affect multiple targets in microbial cell and loss of one of these targets leaves others still responsive (McDonnell and Russell, 1999). This raises the question whether the two fold serial dilution protocol adopted for determination of MIC for antibiotics is a sensitive enough to detect the slight changes in susceptibility expected with biocides. There is a need for the development of standard protocols for the quantitative assessment of biocide induced resistance and cross resistance (SCENIHR, 2009).

$S$. aureus is a popular nosocomial pathogen that presents a therapeutic problem due to its ability to rapidly acquire resistance to frequently used drugs. The objective of the present study was to: first, verify whether MICs can be employed to correlate resistance to biocides and antibiotics in Staphylococcus species; and secondly, to find whether this possible correlation could vary according to isolates' background by comparing MICs for isolates from various sources with the likelihood of exposure to biocides and/or antibiotics.

\section{MATERIALS and METHODS:}

\section{Bacterial isolates}

The study involved r 140
staphylococcal isolates, comprising 47
clinical hospital isolates, 64 hospital
environmental isolates from Zagazig
University Hospitals and 33 non hospital
environmental isolates collected during the
period between May 2005 and June 2006.

The isolates were identified according to Koneman et al (1997).

\section{Antimicrobials and culture media}

Pharmaceutical grades of antibiotics and biocides were obtained from the local pharmaceutical companies. Oxacillin , vancomycin, chlorhexidine $\mathrm{HCl}$, benzalkonium chloride, cetrimide, phenyl mercuric nitrate, ethidium bromide and chlorocresol were obtained from Sigma Chemical Company, St. Louis, Mo, USA. Culture media and oxacillin discs were obtained form from Oxoid, Hampshire, England.

\section{Detection of methicillin resistance}

Detection of methicillin resistance was carried out using oxacillin discs $(1 \mu \mathrm{g})$ according to NCCLS (1993).

\section{Determination of minimum inhibitory concentration (MIC)}

The MICs of antimicrobial agents were determined using agar dilution method according to NCCLS (1997). For biocides, increasing concentrations with constant increments (arithmetic progression) were made in Mueller-Hinton agar instead of twofold serial dilution. The isolates were categorized as antibiotic resistant or sensitive according to NCCL breakpoints. Resistance breakpoints to chlorocresol, benzalkonium chloride, cetrimide, chlorhexidine, phenyl mercuric nitrate, ethidium bromide and povidone-Iodine was assumed by MIC values equal to or greater than $250 \mu \mathrm{g} \mathrm{ml}^{-1}, 5.0 \mu \mathrm{g}$ $\mathrm{ml}^{-1}, 8.0 \mu \mathrm{g} \mathrm{ml}^{-1}, 2.0 \mu \mathrm{g} \mathrm{ml}^{-1}, 0.2 \mu \mathrm{g} \mathrm{ml}^{-1}$, $10.0 \mu \mathrm{g} \mathrm{ml}^{-1}$, and $2500 \mu \mathrm{g} \mathrm{ml}^{-1}$, respectively.

\section{Statistical Analysis}

To investigate the degree of crossresistance between two antimicrobials, Pearson's correlation coefficients (r), which give a measure of the strength of any linear relationship between MIC values (or their $\log _{10}$ derivatives) of the two antimicrobials, 
were calculated using Prism software (GraphPad software, Inc. La Jolla, CA, USA version 5.01) at the same two tailed $\mathrm{P}<0.05$. For an exact relationship, the correlation is 1 or -1 , depending on the relationship and if there is no linear relationship, the correlation tends to zero. MIC data were transformed into $\log _{10}$ MIC and either MIC-MIC, log MICMIC, or log MIC-log MIC combinations for antibiotics and biocides, respectively were used and data were compared for magnitude of the coefficient and discrepancies between them in terms of significance and non significance.

\section{RESULTS:}

\section{Detection of methicillin resistance among Staphylococcus isolates}

Higher percentages of methicillin resistant isolates were found among clinical (31.9\%), and hospital environment isolates $(28.1 \%)$ compared to non hospital environmental isolates $(9.1 \%)$.

\section{Resistance of Staphylococcus isolates to antimicrobial agents}

Relatively higher resistance rates to individual antibiotics and biocides were found among isolates from hospital compared to non hospital isolates (Table 1).

Table 1. Percentage Staphylococcus isolates resistant antibiotics and biocides*

\begin{tabular}{|c|c|c|c|c|}
\hline \multirow{2}{*}{$\begin{array}{l}\text { Antimicrobial } \\
\text { agents }\end{array}$} & \multirow{2}{*}{$\begin{array}{l}\text { Resistance } \\
\text { breakpoint } \\
\text { concentration ( } \mu \mathrm{g} \\
\left.\mathrm{ml}^{-1}\right)\end{array}$} & \multicolumn{3}{|c|}{ Percent of resistant isolates among } \\
\hline & & $\begin{array}{c}\text { Clinical } \\
\text { isolates } \\
n=47\end{array}$ & $\begin{array}{c}\text { hospital } \\
\text { environment } \\
\text { isolates } n=64\end{array}$ & $\begin{array}{l}\text { non-hospital } \\
\text { environment } \\
\text { isolates } n=33\end{array}$ \\
\hline penicillin $\mathrm{G}$ & 0.25 & 70.2 & 53.1 & 36.4 \\
\hline Ampicillin & 0.25 & 70.2 & 46.9 & 36.4 \\
\hline Oxacillin & 4 & 31.9 & 28.1 & 9.1 \\
\hline Vancomycin & 2 & 0 & 0 & 0 \\
\hline Cefepime & 64 & 29.8 & 15.6 & 12.1 \\
\hline Streptomycin & 16 & 31.9 & 42.2 & 36.4 \\
\hline Tetracycline & 16 & 29.8 & 35.9 & 21.2 \\
\hline Gentamicin & 16 & 17 & 9.4 & 3.0 \\
\hline Azithromycin & 8 & 36.2 & 42.2 & 9.1 \\
\hline Ciprofloxacin & 4 & 19.1 & 23.4 & 15.2 \\
\hline Chloramphenicol & 32 & 12.8 & 4.7 & 0 \\
\hline Chlorocresol & 250 & 27.7 & 21.9 & 12.1 \\
\hline Benzalkonium chloride & 4 & 59.6 & 60.9 & 48.5 \\
\hline Cetrimide & 8 & 19.1 & 34.4 & 21.2 \\
\hline Phenyl mercuric nitrate & 0.2 & 51.1 & 59.4 & 39.4 \\
\hline Ethidium bromide & 10 & 12.2 & 25 & 0 \\
\hline Povidone-Iodine & 2500 & 70.2 & 79.7 & 54.5 \\
\hline
\end{tabular}

* Biocide resistance calculated based on the suggested breakpoint concentration

\section{Comparison of susceptibility of isolates to biocides and antibiotics}

Pearson's correlation coefficient (r) between MIC-MIC, MIC-log MIC, and log MIC-log MIC were calculated for individual groups and total isolates. Pearson's coefficients (r) for MIC values and their significance are shown in tables (2-5). In case of discrepancy in the interpretation of significance of correlation between MIC-MIC comparison and each of log MIC-MIC or log MIC-log MIC, it was indicated as superscript. 
Table 2. Pearson's correlation coefficient between antibiotic and biocide MIC for clinical isolates and its significance of correlation

\begin{tabular}{|c|c|c|c|c|c|c|c|}
\hline Antibiotic/biocide & $\mathrm{CC}$ & BKC & CET & CHX & PMN & $\mathrm{PI}$ & EB \\
\hline Penicillin G & $0.64 * * *$ & $0.47 * * *$ & $0.59 * * *$ & $-0.04 \mathrm{NS}$ & $0.21 \mathrm{NS}^{\mathrm{a}}$ & $-0.45 * * \mathrm{~d}$ & $0.43 \mathrm{NS}^{\mathrm{a}}$ \\
\hline Ampicillin & $0.62 * * *$ & $-0.34 * *$ & $0.39 * * *$ & $0.13 \mathrm{NS}$ & $0.23 \mathrm{NS}^{\mathrm{a}}$ & $-0.37 *^{\mathrm{d}}$ & $0.34 \mathrm{NS}^{\mathrm{a}}$ \\
\hline Oxacillin & $0.53 * * *$ & $0.47 * * *$ & $0.54 * * *$ & $0.17 \mathrm{NS}$ & $0.26 \mathrm{NS}$ & $-0.33^{\mathrm{cd}}$ & $0.26 \mathrm{NS}^{\mathrm{ab}}$ \\
\hline Cefepime & $0.64 * * *$ & $0.11^{* *}$ & $0.34 *$ & $0.02 \mathrm{NS}$ & $0.35^{*}$ & $-0.46 * * * *^{\mathrm{d}}$ & $0.27 \mathrm{NS}^{\mathrm{ab}}$ \\
\hline Vancomycin & $-0.29 \mathrm{NS}$ & $0.24 \mathrm{NS}$ & $-0.19 \mathrm{NS}$ & $0.44 \mathrm{NS}^{\mathrm{ab}}$ & $-0.09 \mathrm{NS}$ & $0.29 *^{\mathrm{c}}$ & $-0.14 \mathrm{NS}$ \\
\hline Streptomycin & $0.65 * * *$ & $0.47 * * *$ & $0.50 * * *$ & $-0.17 \mathrm{NS}$ & $0.25 \mathrm{NS}$ & $-0.55 * * *$ cd & $0.37 *$ \\
\hline Gentamicin & $0.45^{* *}$ & $0.53 \mathrm{NS}^{\mathrm{b}}$ & $-0.057 \mathrm{NS}$ & $-0.05 \mathrm{NS}$ & $0.14 \mathrm{NS}$ & $-0.35^{* \mathrm{~cd}}$ & $-0.08 * * * \mathrm{~cd}$ \\
\hline Tetracycline & $0.39 * * \mathrm{~d}$ & $0.06 \mathrm{NS}$ & $0.022 \mathrm{NS}$ & $-0.04 \mathrm{NS}$ & $0.35 \mathrm{NS}$ & $-0.35^{\text {cd }}$ & $0.09 \mathrm{NS}$ \\
\hline Azithromycin & $0.32 *$ & $0.09 \mathrm{NS}^{\mathrm{ab}}$ & $0.17 \mathrm{NS}^{\mathrm{ab}}$ & $0.09 \mathrm{NS}$ & $0.17 \mathrm{NS}$ & $-0.24 \mathrm{NS}$ & $0.21 \mathrm{NS}^{\mathrm{ab}}$ \\
\hline Chloramphenicol & $0.48 * * *$ & $0.516 \mathrm{NS}$ & $-0.08 \mathrm{NS}$ & $-0.07 \mathrm{NS}$ & $0.24 \mathrm{NS}$ & $-0.41 * *$ cd & $-0.12 *^{\mathrm{cd}}$ \\
\hline Ciprofloxacin & $0.13 \mathrm{NS}^{\mathrm{ab}}$ & $-0.34 * * *$ & $0.59 * * *$ & $-0.03 \mathrm{NS}$ & $-0.10 \mathrm{NS}$ & $-0.11 N S$ & $0.85 \mathrm{NS}^{\mathrm{ab}}$ \\
\hline
\end{tabular}

* significant correlation( $\mathrm{p}$ between 0.01 and 0.05 )

*** very significant correlation ( $\mathrm{p}$ is less than 0.01 )

*** extremely significant correlation ( $\mathrm{p}$ is less than 0.001 )

a significant with antibiotic log MIC versus biocide MIC

${ }^{\mathrm{b}}$ significant with antibiotic $\log$ MIC versus biocide $\log$ MIC

${ }^{\mathrm{c}}$ non-significant with antibiotic log MIC versus biocide MIC

${ }^{\mathrm{d}}$ non-significant with antibiotic $\log$ MIC versus biocide log MIC

Table 3. Pearson's correlation coefficient between antibiotic and biocide MIC for Hospital environmental isolates and its significance of correlation

\begin{tabular}{|c|c|c|c|c|c|c|c|}
\hline Antibiotic/biocide & $\mathrm{CC}$ & $\mathrm{BKC}$ & CET & CHX & PMN & $\mathrm{PI}$ & EB \\
\hline Penicillin G & $0.41 * * *$ & $0.54 * * *$ & $0.64 * * *$ & $-0.10 \mathrm{NS}$ & $0.26^{* c}$ & $0.13 \mathrm{NS}$ & 0.50 *** \\
\hline Ampicillin & $0.38 * *$ & $0.46 * * *$ & $0.55 * * *$ & $-0.06 \mathrm{NS}$ & $0.23 \mathrm{NS}$ & $0.09 \mathrm{NS}$ & $0.44 * * *$ \\
\hline Oxacillin & $0.42^{* * *}$ & $0.57 * * *$ & $0.66 * * *$ & $0.031 \mathrm{NS}$ & $0.33 * *$ & $0.08 \mathrm{NS}$ & $0.56^{* * *}$ \\
\hline Cefepime & $0.446 * * *$ & $0.38 * *$ & $0.48 * * *$ & $-0.03 \mathrm{NS}$ & $0.33 * *$ & $-0.03 \mathrm{NS}$ & $0.36^{* *}$ \\
\hline Vancomycin & $0.27 *^{\mathrm{cd}}$ & $0.22 \mathrm{NS}$ & $0.33 * *$ & $-0.15 N S$ & $0.18 \mathrm{NS}$ & $0.15 \mathrm{NS}$ & $0.19 \mathrm{NS}^{\mathrm{b}}$ \\
\hline Streptomycin & $0.57 * * *$ & $0.63 * * *$ & $0.64 * * *$ & $-0.06 \mathrm{NS}$ & $0.34 * *$ & $0.08 \mathrm{NS}$ & $0.57 * * *$ \\
\hline Gentamicin & $0.39 * *$ & $0.32 * *$ & $0.13 \mathrm{NS}^{\mathrm{ab}}$ & $0.01 \mathrm{NS}$ & $0.19 \mathrm{NS}$ & $-0.18 \mathrm{NS}$ & $0.33^{* *}$ \\
\hline Tetracycline & $0.36 \mathrm{NS}$ & $0.17 \mathrm{NS}^{\mathrm{ab}}$ & $-0.04 N S$ & $-0.04 \mathrm{NS}$ & $0.15 \mathrm{NS}$ & $-0.17 \mathrm{NS}$ & $0.02 \mathrm{NS}$ \\
\hline Azithromycin & $0.27 *$ & $0.32 * *$ & $0.30 *$ & $0.12 \mathrm{NS}$ & $0.17 \mathrm{NS}^{\mathrm{b}}$ & $-0.22 \mathrm{NS}$ & $0.31 *$ \\
\hline Chloramphenicol & $0.35 * *$ & $0.39 * *$ & $0.22 \mathrm{NS}^{\mathrm{ab}}$ & $0.14 \mathrm{NS}$ & $0.18 \mathrm{NS}$ & $-0.17 \mathrm{NS}$ & $0.51 * * *$ \\
\hline Ciprofloxacin & $0.22 \mathrm{NS}^{\mathrm{a}}$ & $0.19 \mathrm{NS}^{\mathrm{ab}}$ & $0.33 * *$ & $-0.06 \mathrm{NS}$ & $0.27 *$ & $0.09 \mathrm{NS}$ & $0.22 \mathrm{NS}^{\mathrm{ab}}$ \\
\hline
\end{tabular}

* significant correlation( $\mathrm{p}$ between 0.01 and 0.05 )

${ }^{* *}$ very significant correlation ( $\mathrm{p}$ is less than 0.01 )

*** extremely significant correlation ( $\mathrm{p}$ is less than 0.001 )

${ }^{a}$ significant with antibiotic $\log$ MIC versus biocide MIC

${ }^{\mathrm{b}}$ significant with antibiotic $\log$ MIC versus biocide $\log$ MIC

${ }^{c}$ non-significant with antibiotic log MIC versus biocide MIC

${ }^{d}$ non-significant with antibiotic log MIC versus biocide log MIC 
Table 4. Pearson's correlation coefficient between antibiotic and biocide MIC for environmental isolates and its significance of correlation

\begin{tabular}{|c|c|c|c|c|c|c|c|}
\hline Antibiotic/biocide & $\mathrm{CC}$ & $\mathrm{BKC}$ & CET & CHX & PMN & PI & EB \\
\hline Penicillin G & $0.01 \mathrm{NS}$ & $0.35^{* \mathrm{~cd}}$ & $0.86^{* * * *}$ & $-0.16 \mathrm{NS}$ & $0.12 \mathrm{NS}$ & $0.02 \mathrm{NS}$ & $0.23 \mathrm{NS}$ \\
\hline Ampicillin & $0.12 \mathrm{NS}$ & $0.32 \mathrm{NS}$ & $0.82 * * *$ & $-0.21 \mathrm{NS}$ & $0.09 \mathrm{NS}$ & $0.04 \mathrm{NS}$ & $0.24 \mathrm{NS}$ \\
\hline Oxacillin & $0.34 \mathrm{NS}$ & $0.61 * * *$ & $0.97 * * *$ & $-0.14 N S$ & $0.29 \mathrm{NS}^{\mathrm{ab}}$ & $-0.09 \mathrm{NS}^{\mathrm{ab}}$ & $0.16 \mathrm{NS}$ \\
\hline Cefepime & $0.28 \mathrm{NS}$ & $0.52^{* *} \mathrm{~d}$ & $0.97 * * *$ & $-0.14 N S$ & $0.25 \mathrm{NS}$ & $-0.11 \mathrm{NS}$ & $0.16 \mathrm{NS}$ \\
\hline Vancomycin & $0.17 \mathrm{NS}$ & $-0.12 \mathrm{NS}$ & $-0.04 \mathrm{NS}$ & $0.12 \mathrm{NS}$ & $-0.29 \mathrm{NS}$ & $0.09 \mathrm{NS}$ & $0.04 \mathrm{NS}$ \\
\hline Streptomycin & $0.08 \mathrm{NS}$ & $0.25 \mathrm{NS}$ & $0.45^{* *}$ & $-0.24 \mathrm{NS}$ & $0.02 \mathrm{NS}$ & $-0.21 \mathrm{NS}$ & $0.35^{*}$ \\
\hline Gentamicin & $0.27 \mathrm{NS}$ & $0.43^{\text {cd }}$ & $0.91 * * *$ & $-0.09 \mathrm{NS}$ & $0.20 \mathrm{NS}$ & $-0.21 \mathrm{NS}$ & $0.12 \mathrm{NS}$ \\
\hline Tetracycline & $-0.23 \mathrm{NS}$ & $0.34 \mathrm{NS}$ & $0.09 \mathrm{NS}$ & $-0.32 \mathrm{NS}$ & $0.19 \mathrm{NS}$ & $0.04 \mathrm{NS}$ & $0.11 \mathrm{NS}$ \\
\hline Azithromycin & $-0.19 \mathrm{NS}$ & $0.39 *$ & $0 . .017 \mathrm{NS}$ & $-0.14 N S$ & $0.31 \mathrm{NS}$ & $-0.08 \mathrm{NS}$ & $-0.11 \mathrm{NS}$ \\
\hline Chloramphenicol & $-0.23 \mathrm{NS}$ & $0.51 * *$ & $0.39 \mathrm{NS}^{\mathrm{b}}$ & $-0.47 * * \mathrm{~cd}$ & $0.27 \mathrm{NS}$ & $-0.11 \mathrm{NS}$ & $0.07 \mathrm{NS}$ \\
\hline Ciprofloxacin & $-0.27 \mathrm{NS}$ & $0.35^{*}$ & $0.12 \mathrm{NS}^{\mathrm{b}}$ & $-0.29 \mathrm{NS}$ & $0.16 \mathrm{NS}$ & $0.06 \mathrm{NS}$ & $0.15 \mathrm{NS}$ \\
\hline
\end{tabular}

* significant correlation(p between 0.01 and 0.05 )

** very significant correlation ( $\mathrm{p}$ is less than 0.01 )

extremely significant correlation ( $\mathrm{p}$ is less than 0.001 )

a significant with antibiotic log MIC versus biocide MIC

${ }^{\mathrm{b}}$ significant with antibiotic $\log$ MIC versus biocide log MIC

${ }^{c}$ non-significant with antibiotic log MIC versus biocide MIC

${ }^{d}$ non-significant with antibiotic log MIC versus biocide log MIC

Table 5. Pearson's correlation coefficient between antibiotic and biocide MIC for combined isolates and its significance of correlation

\begin{tabular}{|c|c|c|c|c|c|c|c|}
\hline Antibiotic/biocide & $\mathrm{CC}$ & $\mathrm{BKC}$ & CET & $\mathrm{CHX}$ & PMN & PVI & EB \\
\hline Penicillin G & $0.44 * * *$ & $0.52 * * *$ & $0.64 * * *$ & $-0.05 \mathrm{NS}$ & $0.22 * *$ & $-0.08 \mathrm{NS}$ & 0.46 *** \\
\hline Ampicillin & $0.44 * * *$ & $0.41 * * *$ & $0.46^{* * *}$ & $0.08 \mathrm{NS}$ & $0.21 *$ & $-0.11 \mathrm{NS}$ & $0.36 * * *$ \\
\hline Oxacillin & $0.41 * * *$ & $0.52 * * *$ & $0.63 * * *$ & $0.12 \mathrm{NS}$ & $0.29 * * *$ & $-0.10 \mathrm{NS}$ & $0.39 * * *$ \\
\hline Cefepime & $0.46 * * *$ & $0.37 * * *$ & $0.42 * * *$ & $0.03 \mathrm{NS}$ & $0.31 * * *$ & $-0.18^{*}$ & $0.32 * * *$ \\
\hline Vancomycin & $0.00 \mathrm{NS}$ & $-0.04 \mathrm{NS}$ & $0.07 \mathrm{NS}$ & $0.15 \mathrm{NS}$ & $-0.02 \mathrm{NS}$ & $0.19 *$ & $0.06 \mathrm{NS}$ \\
\hline Streptomycin & $0.51 * * *$ & $0.50 * * *$ & $0.555 * * *$ & $-0.11 \mathrm{NS}$ & $0.23 * *$ & $-0.21 *$ & $0.43 * * *$ \\
\hline Gentamicin & $0.31 * * *$ & $0.11 \mathrm{NS}$ & $0.00 \mathrm{NS}$ & $-0.00 \mathrm{NS}$ & $0.11 \mathrm{NS}^{\mathrm{ab}}$ & $-0.21 *$ & $0.03 \mathrm{NS}$ \\
\hline Tetracycline & $0.19^{* \mathrm{~cd}}$ & $0.17 *$ & $0.01 \mathrm{NS}^{\mathrm{b}}$ & $-0.04 \mathrm{NS}$ & $0.14 \mathrm{NS}^{\mathrm{b}}$ & $-0.19 *$ & $0.06 \mathrm{NS}$ \\
\hline Azithromycin & $0.23 * *$ & $0.28 * * *$ & $0.25^{* *}$ & $0.12 \mathrm{NS}$ & $0.19 *$ & $-0.15 \mathrm{NS}$ & $0.30 * * *$ \\
\hline chloramphenicol & $0.344 * *$ & $0.19 *$ & $0.06 \mathrm{NS}$ & $0.03 \mathrm{NS}$ & $0.19 *$ & $-0.24 * *$ cd & $0.17 *^{\mathrm{cd}}$ \\
\hline Ciprofloxacin & $0.14 \mathrm{NS}^{\mathrm{ab}}$ & $0.33 * * *$ & $0.42 * * *$ & $-0.03 N S$ & $0.12 \mathrm{NS}^{\mathrm{ab}}$ & $0.02 \mathrm{NS}$ & $0.45^{* * *}$ \\
\hline
\end{tabular}

* significant correlation(p between 0.01 and 0.05 )

*** very significant correlation ( $\mathrm{p}$ is less than 0.01 )

* extremely significant correlation ( $\mathrm{p}$ is less than 0.001 )

${ }^{a}$ significant with antibiotic $\log$ MIC versus biocide MIC

${ }^{\mathrm{b}}$ significant with antibiotic $\log$ MIC versus biocide log MIC

${ }^{c}$ non-significant with antibiotic log MIC versus biocide MIC

${ }^{d}$ non-significant with antibiotic log MIC versus biocide log MIC

Further analyses for MIC data based on comparing the percentages of antibiotic resistant isolates among biocide-susceptible and biocide-resistant ones are presented in tables (6-12). 
Table 6. The percentage of antibiotic resistant isolates among chlorhexidine susceptible and resistant isolates.

\begin{tabular}{l|c|c|c|c|c|c|c|c}
\hline \multirow{2}{*}{$\begin{array}{l}\text { Type of isolates } \\
\text { antibiotic }\end{array}$} & \multicolumn{2}{|c|}{ Clinical $(\mathrm{n}=47)$} & \multicolumn{2}{c|}{ Hospital env $(\mathrm{n}=64)$} & \multicolumn{2}{c|}{ Non hosp env $(\mathrm{n}=33)$} & \multicolumn{2}{c}{ Total $(\mathrm{n}=144)$} \\
\cline { 2 - 9 } & $\mathrm{S}=37$ & $\mathrm{R}=10$ & $\mathrm{~S}=53$ & $\mathrm{R}=11$ & $\mathrm{~S}=33$ & $\mathrm{R}=0$ & $\begin{array}{l}\mathrm{S}=123 \\
(85.4 \%)\end{array}$ & $\mathrm{R}=21$ \\
\hline Penicillin & 86.5 & 10 & 62.3 & 9.1 & 12.1 & 0.0 & 62.6 & 9.5 \\
Ampicillin & 86.5 & 10 & 54.7 & 9.1 & 36.4 & 0.0 & 59.3 & 9.5 \\
Oxacillin & 37.8 & 10 & 32.1 & 9.1 & 9.1 & 0.0 & 27.6 & 9.5 \\
Cefepime & 35.1 & 10 & 17.0 & 9.1 & 12.1 & 0.0 & 21.1 & 9.5 \\
Streptomycin & 40.5 & 0.0 & 43.4 & 36.4 & 36.4 & 0.0 & 40.6 & 19.0 \\
Tetracycline & 29.7 & 30 & 43.4 & 0.0 & 21.2 & 0.0 & 27.6 & 14.3 \\
Gentamicin & 21.6 & 0.0 & 11.3 & 0.0 & 3.0 & 0.0 & 12.2 & 4.8 \\
Azithromycin & 40.5 & 20 & 43.4 & 36.4 & 9.1 & 0.0 & 33.3 & 28.6 \\
Ciprofloxacin & 24.3 & 0.0 & 26.4 & 9.1 & 15.1 & 0.0 & 22.8 & 4.8 \\
Chloramphenicol & 16.2 & 0.0 & 3.8 & 9.1 & 0 & 0.0 & 6.5 & 4.8 \\
\hline
\end{tabular}

Table 7. The percentage of antibiotic resistant isolates among Chlorocresol susceptible and resistant isolates.

\begin{tabular}{|c|c|c|c|c|c|c|c|c|}
\hline \multirow{2}{*}{$\begin{array}{l}\text { Type of isolates } \\
\text { Antibiotic }\end{array}$} & \multicolumn{2}{|c|}{ Clinical } & \multicolumn{2}{|c|}{ Hospital env } & \multicolumn{2}{|c|}{ Non hosp env } & \multicolumn{2}{|c|}{ Total } \\
\hline & $\mathrm{S}=34$ & $\mathrm{R}=13$ & $\mathrm{~S}=50$ & $\mathrm{R}=14$ & $\mathrm{~S}=29$ & $\mathrm{R}=4$ & $\begin{array}{l}S=113 \\
(78.47 \%)\end{array}$ & $\mathrm{R}=31$ \\
\hline Penicillin & 61.8 & 92.3 & 48 & 71.4 & 34.5 & 50 & 48.7 & 74 \\
\hline Ampicillin & 61.8 & 92.3 & 40 & 71.4 & 34.5 & 50 & 45.1 & 74 \\
\hline Oxacillin & 8.8 & 92.3 & 16 & 71.4 & 6.9 & 25 & 11.5 & 71 \\
\hline Cefepime & 5.9 & 92.3 & 6 & 50 & 10.3 & 25 & 7.1 & 64.5 \\
\hline Streptomycin & 11.8 & 84.6 & 26 & 100 & 34.5 & 50 & $(23.9$ & 87.1 \\
\hline Tetracycline & 20.6 & 53.8 & 36 & 35.7 & 20.7 & 25 & 27.4 & 41.9 \\
\hline Gentamicin & 0.0 & 61.5 & 2 & 35.7 & 0.0 & 25 & 0.9 & 45.2 \\
\hline Azithromycin & 14.7 & 92.3 & 34 & 71.4 & 6.9 & 25 & $(21.2$ & 71 \\
\hline Ciprofloxacin & 2.9 & 61.5 & 14 & 57.1 & 17.2 & 0.0 & 11.5 & 51.6 \\
\hline Chloramphenicol & 0.0 & 46.2 & 0.0 & 21.4 & 0.0 & 0.0 & 0.0 & 29 \\
\hline
\end{tabular}

Table 8. The percentage of antibiotic resistant isolates among Phenyl mercuric nitrate susceptible and resistant isolates.

\begin{tabular}{l|c|c|c|c|c|c|c|c}
\hline $\begin{array}{l}\text { Type of isolates } \\
\text { Antibiotic }\end{array}$ & \multicolumn{2}{|l|}{ Clinical } & \multicolumn{2}{l|}{ Hospital env } & \multicolumn{2}{l|}{ Non hosp env } & \multicolumn{2}{l}{ Total } \\
\cline { 2 - 9 } & $\mathrm{S}=23$ & $\mathrm{R}=24$ & $\mathrm{~S}=26$ & $\mathrm{R}=38$ & $\mathrm{~S}=20$ & $\mathrm{R}=13$ & $\begin{array}{l}\mathrm{S}=69 \\
(47.9 \%)\end{array}$ & $\mathrm{R}=75$ \\
\hline Penicillin & 60.9 & 79.2 & 46.2 & 57.9 & 35 & 38.5 & 47.8 & 61.3 \\
Ampicillin & 60.9 & 79.2 & 38.5 & 52.6 & 35 & 38.5 & 44.9 & 58.7 \\
Oxacillin & 17.4 & 45.8 & 15.4 & 36.8 & 0.0 & 23.1 & 11.6 & 37.3 \\
Cefepime & 13 & 45.8 & 0.0 & 26.3 & 5 & 23.1 & 5.8 & 32 \\
Streptomycin & 26.1 & 37.5 & 26.9 & 52.6 & 35 & 38.5 & 30 & 45.3 \\
Tetracycline & 30.7 & 29.2 & 30.8 & 39.5 & 15 & 30.8 & 26.1 & 34.7 \\
Gentamicin & 8.7 & 25 & 3.8 & 13.2 & 0.0 & 7.7 & 4.3 & 16 \\
Azithromycin & 21.7 & 50 & 26.9 & 52.6 & 0.0 & 23.1 & 17.4 & 46.7 \\
Ciprofloxacin & 8.7 & 29.2 & 3.8 & 36.8 & 10 & 23.1 & 6.7 & 32 \\
Chloramphenicol & 4.3 & 20.8 & 0.0 & 7.9 & 0.0 & 0.0 & 1.44 & 10.7 \\
\hline
\end{tabular}


Table 9. The percentage of antibiotic resistant isolates among benzalkonium chloride susceptible and resistant isolates.

\begin{tabular}{l|c|c|c|c|c|c|c|c}
\hline $\begin{array}{l}\text { Type of isolates } \\
\text { Antibiotic }\end{array}$ & \multicolumn{2}{|l|}{ Clinical isolates } & \multicolumn{2}{l|}{ Hospital env } & \multicolumn{2}{l|}{ Non hosp env } & \multicolumn{2}{l}{ Total } \\
\cline { 2 - 9 } & $\mathrm{S}=19$ & $\mathrm{R}=28$ & $\mathrm{~S}=25$ & $\mathrm{R}=39$ & $\mathrm{~S}=17$ & $\mathrm{R}=16$ & $\mathrm{~S}=61$ & $\mathrm{R}=83$ \\
& & & & & & & $42.4 \%)$ & \\
\hline Penicillin G & 31.6 & 96.4 & 48 & 56.4 & 41.2 & 31.2 & 41 & 65.1 \\
Ampicillin & 31.6 & 96.4 & 36 & 53.8 & 41.2 & 31.2 & 36.1 & 63.8 \\
Oxacillin & 5.3 & 50 & 8 & 41 & 0.0 & 18.7 & 4.9 & 39.7 \\
Cefepime & 0.0 & 50 & 0.0 & 25.6 & 5.9 & 18.7 & 1.6 & 32.5 \\
Streptomycin & 15.8 & 42.9 & 12 & 61.5 & 29.4 & 43.7 & 18 & 51.8 \\
Tetracycline & 31.6 & 28.6 & 24 & 43.6 & 17.6 & 25 & 24.6 & 34.9 \\
Gentamicin & 0.0 & 28.6 & 0.0 & 15.4 & 0.0 & 6.2 & 0.0 & 18.1 \\
Azithromycin & 21.1 & 46.4 & 28 & 51.3 & 0.0 & 8.7 & 18 & 43.4 \\
Ciprofloxacin & 0.0 & 32.1 & 16 & 28.2 & 11.8 & 18.7 & 9.8 & 27.7 \\
Chloramphenicol & 0.0 & 21.4 & 0.0 & 7.7 & 0.0 & 0.0 & 0.0 & 10.8 \\
\hline
\end{tabular}

Table 10 . The percentage of antibiotic resistant isolates among cetrimide susceptible and resistant isolates.

\begin{tabular}{l|c|c|c|c|c|c|c|c}
\hline $\begin{array}{l}\text { Type of isolates } \\
\text { Antibiotic }\end{array}$ & \multicolumn{2}{l|}{ Clinical isolates } & \multicolumn{2}{l|}{ Hospital env } & \multicolumn{2}{l|}{ Non hosp env } & \multicolumn{2}{l}{ Total } \\
\cline { 2 - 9 } & $\mathrm{S}=38$ & $\mathrm{R}=9$ & $\mathrm{~S}=42$ & $\mathrm{R}=22$ & $\mathrm{~S}=26$ & $\mathrm{R}=7$ & $\begin{array}{l}\mathrm{S}=106 \\
(73.6 \%)\end{array}$ & $\mathrm{R}=38$ \\
\hline Penicillin & 32.5 & 88.9 & 45.2 & 68.2 & 26.9 & 71.4 & 48.1 & 73.7 \\
Ampicillin & 32.5 & 88.9 & 35.7 & 68.2 & 26.9 & 71.4 & 44.3 & 73.7 \\
Oxacillin & 18.4 & 88.9 & 16.7 & 50 & 0.0 & 42.9 & 13.2 & 57.9 \\
Cefepime & 15.8 & 88.9 & 7.1 & 31.8 & 3.8 & 42.9 & 9.4 & 47.4 \\
Streptomycin & 18.4 & 88.9 & 35.7 & 54.5 & 19.2 & 100 & 25.5 & 71 \\
Tetracycline & 28.9 & 33.3 & 38.1 & 31.8 & 11.5 & 57.1 & 28.3 & 36.8 \\
Gentamicin & 13.2 & 33.3 & 4.8 & 18.2 & 0.0 & 14.3 & 6.6 & 21 \\
Azithromycin & 26.3 & 77.8 & 23.8 & 77.3 & 3.8 & 28.6 & 19.8 & 68.4 \\
Ciprofloxacin & 13.2 & 44.4 & 14.3 & 40.9 & 3.8 & 57.1 & 11.3 & 44.7 \\
Chloramphenicol & 10.5 & 22.2 & 2.4 & 9.1 & 0.0 & 0.0 & 4.7 & 7.9 \\
\hline
\end{tabular}

Table 11. The percentage of antibiotic resistant isolates among ethidium bromide susceptible and resistant isolates.

\begin{tabular}{|c|c|c|c|c|c|c|c|c|}
\hline Type of isolates & Clinical & lates & Hospita & & Non ho & & Total & \\
\hline antibiotic & $S=41$ & $\mathrm{R}=6$ & $\mathrm{~S}=48$ & $\mathrm{R}=16$ & $\mathrm{~S}=33$ & $\begin{array}{l}\mathrm{R} \\
(0)\end{array}$ & $\begin{array}{l}S=122 \\
(84 \%)\end{array}$ & $\mathrm{R}=22$ \\
\hline Penicillin & 68.3 & 83.3 & 45.8 & 75 & 36.4 & 0.0 & 50.8 & 77.3 \\
\hline Ampicillin & 68.3 & 83.3 & 37.5 & 75 & 36.4 & 0.0 & 47.5 & 77.3 \\
\hline Oxacillin & 24.4 & 83.3 & 18.8 & 56.3 & 9.1 & 0.0 & 18 & 63.6 \\
\hline Cefepime & 22 & 83.3 & 6.3 & 43.8 & 12.1 & 0.0 & 13.9 & 54.5 \\
\hline Streptomycin & 22 & 100 & 33.3 & 68.8 & 36.4 & 0.0 & 30.3 & 77.3 \\
\hline Tetracycline & 26.8 & 50 & 33.3 & 43.8 & 21.2 & 0.0 & 27.9 & 45.4 \\
\hline Gentamicin & 14.6 & 33.3 & 6.3 & 18.8 & 3 & 0.0 & 8.2 & 22.7 \\
\hline Azithromycin & 29.3 & 83.3 & 33.3 & 68.8 & 9.1 & 0.0 & 25.4 & 72.7 \\
\hline Ciprofloxacin & 12.2 & 66.7 & 14.6 & 50 & 15.1 & 0.0 & 13.9 & 54.5 \\
\hline Chloramphenicol & 14.6 & 0.0 & 2.1 & 12.5 & 0.0 & 0.0 & 5.7 & 9.1 \\
\hline
\end{tabular}


Table 12. The percentage of antibiotic resistant isolates among povidone-iodine susceptible and resistant isolates.

\begin{tabular}{l|c|c|c|c|c|c|c|c}
\hline Type of isolates & \multicolumn{2}{l|}{ Clinical isolates } & \multicolumn{2}{l|}{ Hospital env } & \multicolumn{2}{l|}{ Non hosp env } & \multicolumn{2}{l}{ Total } \\
\hline antibiotic & $\mathrm{S}=14$ & $\mathrm{R}=33$ & $\mathrm{~S}=12$ & $\mathrm{R}=51$ & $\mathrm{~S}=15$ & $\mathrm{R}=18$ & $\mathrm{~S}=41$ & $\begin{array}{l}\mathrm{R}=102 \\
(71.5)\end{array}$ \\
\hline Penicillin & 57.1 & 9.1 & 25 & 16.9 & 53.3 & 16.7 & 46.3 & 14.7 \\
Ampicillin & 57.1 & 15.2 & 25 & 16.9 & 53.3 & 27.8 & 46.3 & 18.6 \\
Oxacillin & 64.3 & 18.2 & 50 & 22.6 & 60 & 33.3 & 58.5 & 23.5 \\
Cefepime & 57.1 & 18.2 & 25 & 11.3 & 53.3 & 33.3 & 46.3 & 17.6 \\
Streptomycin & 57.1 & 21.2 & 50 & 39.6 & 53.3 & 38.9 & 53.7 & 34.3 \\
Tetracycline & 42.9 & 21.2 & 33.3 & 32 & 40 & 38.9 & 39 & 30.3 \\
Gentamicin & 50 & 3 & 16.7 & 7.5 & 46.7 & 5.6 & 39 & 5.8 \\
Azithromycin & 85.7 & 15.2 & 75 & 33.9 & 80 & 27.8 & 80.5 & 27.4 \\
Ciprofloxacin & 35.7 & 12.1 & 33.3 & 20.7 & 33.3 & 22.2 & 34.1 & 18.6 \\
Chloramphenicol & 35.7 & 3 & 8.3 & 1.8 & 33.3 & 5.6 & 26.8 & 2.9 \\
\hline
\end{tabular}

Table 13. Comparison of the number of discrepancies in interpretation of significance and non significance for correlation between antibiotic and biocide resistance using MIC-MIC, $\log$ MIC-MIC, and $\log$ MIC-log MIC

\begin{tabular}{lcccccc}
\hline Isolate type & \multicolumn{7}{c}{ Comparison of } \\
\hline & \multicolumn{2}{c}{ MIC-MIC } & \multicolumn{2}{c}{ Log MIC-MIC } & \multicolumn{2}{c}{ Log MIC-log MIC } \\
& NS odds & S odds & NS odds & S odds & NS odds & S odds \\
Clinical isolates & 5 & - & - & 2 & 3 & 1 \\
Hospital env isolates & 5 & 1 & 1 & 1 & - & 2 \\
Environmental isolates & 1 & 2 & - & - & - & 1 \\
Combined isolates & 8 & 4 & - & 1 & - & 4 \\
\hline
\end{tabular}

\section{DISCUSSION:}

Much concern has been raised on the role of widespread use of biocides in various fields including healthcare setting on the development of antibiotic (emergence or selection) resistance among microbes (Russell et al., 1999; Levy 2002; Sheldon, 2005; Weber and Rutala, 2006). The present study aimed to investigate the antibiotic and biocide resistance profiles for staphylococcal isolates from hospital and non hospital sources and to find out any correlation (or cross resistance) between resistances to either agent.

The study focused on Staphylococcus species, as one of the most common pathogens in clinical setting with reputation of rapidly developing resistance to most chemotherapeutic antimicrobial agents
(Johnson et al., 2003; Huang et al., 2007). The approach adopted in the present study was to recover staphylococcal isolates from various sources with likelihood of exposure to antibiotics, biocides, or both, if any, and to compare their susceptibility to selected group of antibiotics and biocides.

In general, the study clearly demonstrated higher methicillin and other antibiotic resistance rates among isolates from clinical and hospital environmental settings compared to the non hospital isolates. This was quite evident for the $\beta$-lactam antibiotics, azithromycin, and gentamicin, but not with streptomycin, tetracycline, and ciprofloxacin, probably reflecting the infrequent use of the first two and the widespread use of the latter. The relatively higher rates in resistance to 
antibiotics and biocides among hospital isolates could in part be attributed to the high proportion of multi drug resistant methicillin resistant isolates that prevail in hospital setting (Al-Masaudi, et al, 1991; Cookson et al, 1991; Akimitsu et al., 1999; Irizarry et al, 1996; Lambert, 2004; Suller and Russell, 1999).

As they have more subtle targets, resistance to antibiotics is reflected as a distinctive rise in the MICs that could be easily detected by two fold serial dilution procedure for determination of MIC. However, this dilution pattern may not be suitable for detection of the slight changes in MICs attributed to loss or reduced sensitivity to one of the multiple targets of the given biocide. Therefore, arithmetic rather than geometric progression was used for determination of MIC of biocides, and subsequently comparison based on the log MIC values that seem to be more reasonable and was proposed.

Pearson's correlation coefficients between MIC-MIC, log MIC-MIC, and log MIC-log MIC for antibiotic- biocide pairs were determined and compared. The three values for each antibiotic-biocide pair were compared for agreements and discrepancies (odds) with respect to the significance or non significance, of the potential correlation (table 13). Out of the three comparisons, log MIC for antibiotic versus MIC for biocide seemed to be the most representative for trends with the least number of odds, followed by $\log$ MIC-log MIC comparison and finally came the MIC-MIC comparison. Yet, all three comparisons, with few exceptions, confirmed significant correlations.

For hospitals' isolates and except for chlorhexidine and povidone-iodine and occasionally with phenyl mercuric nitrate, a positive correlation was found between resistance to the tested biocide and most of the tested antibiotics. For non-hospital isolates only cetrimide resistance correlated with antibiotic resistance. Collectively for the combined isolates, however, the positive correlation was found between antibiotics and biocides except for chlorhexidine and povidone-iodine.

Another way to correlate biocide resistance with antibiotic resistance was to compare the percentage of isolates resistant to individual antibiotics for biocide resistant and susceptible isolates. Except for chlorhexidine and povidone-iodine, the frequency of antibiotic resistance was higher among biocide resistant than biocide susceptible isolates. Surprisingly, the frequencies of antibiotic resistance were higher among the biocide sensitive than in the biocide resistant isolates for chlorhexidine or povidone-iodine.

In conclusion, the current study demonstrates high frequency of resistance of Staphylococcus isolates from hospital sources to some common antimicrobial agents and a positive correlation between biocides and antibiotics resistance was proven. Exceptions from this were with chlorhexidine and povidone-iodine, where resistance did not correlate with resistance to antibiotics. The study demonstrates the validity of the proposed approch for correlating antibiotic and biocide resistance based on MIC data and also demonstrates high antibiotic and biocide resistance in hospital setting.

\section{REFERENCES}

Al-Masaudi SB, Russell AD, and Day MJ (1991) Comparative sensitivity to antibiotics and biocides of methicillinresistant Staphylococcus aureus strains isolated from Saudi Arabia and Great Britain. J Appl Bacteriol 71, 331-338.

Akimitsu N, Hamamoto H, Inoue R, Shoji M, Akamine A, Takemori K, Hamasaki N, and Sekimizu K. (1999) Increase in resistance of methicillin resistant Staphylococcus aureus to B-lactams caused by mutations conferring resistance to benzalkonium chloride, a disinfectant widely used in hospitals. 
Antimicrob Agents Chemother 43, 3042-3043.

Carson RT, Larson E, Levy SB, Marshall BM, and Aiello AE (2008) Use of antibacterial consumer products containing quaternary ammonium compounds and drug resistance in the community. J Antimicrob Chemother 62, 1160-1162.

Cookson BD, Bolton MC, and Platt JH (1991) Chlorhexidine resistance in methicillinresistant Staphylococcus aureus or just an elevated MIC? An in vitro and in vivo assessment. Antimicrob Agents Chemother 35, 1997-2002.

Fraise AP (2002) Biocide abuse and antimicrobial resisance- a cause for concern? J Antimicrob Chemother 49, 11-12.

Huang SS, Lee SC, Lee N, See LC, Tsai MH, and Shieh WB (2007) Comparison of in vitro activities of levofloxacin, ciprofloxacin, ceftazidime, cefepime, imipenem, and piperacillin-tazobactam against aerobic bacterial pathogens from patients with nosocomial infections. J Microbiol Immunol Infect 40,134-140.

Irizarry L, Merlin T, Rupp J, and Griffith J (1996) Reduced susceptibility of methicillin resistant Staphylococcus aureus to cetylpyridinium chloride and chlorhexidine. Chemotherapy 42: 248252.

Jones RD (1999). Bacterial resistance and topical antimicrobial wash products. Amer J Infect Control 27:351-363.

Johnson AP, Henwood C, Mushtaq S, James $\mathrm{D}$, Warner $\mathrm{M}$, and Livermore $\mathrm{D} \mathrm{M}$ (2003) Susceptibility of Gram-positive bacteria from ICU patients in UK hospitals to antimicrobial agents. J Hosp Infect 54,179-87.

Koneman EW, Allen SD, Janda WM, Schreckenberger PC, and Winn WC (1997) Staphylococci and related organisms. In Colour Atlas And
Textbook Of Diagnostic Microbiology, $5^{\text {th }}$ Ed. J. B. Lippincott company, Philadelphia, 539 - 566.

Lambert RJ (2004) Comparative analysis of antibiotic and antimicrobial biocide susceptibility data in clinical isolates of methicillin-sensitive Staphylococcus aureus, methicillin-resistant Staphylococcus aureus and Pseudomonas aeruginosa between 1989 and 2000. J Appl Microbiol 97, 699-711.

Levy SB (2002) Active efflux, a common mechanism for biocide and antibiotic resistance. J Appl Microbiol 92, 65s$71 \mathrm{~s}$.

Maillard JY (2007) Bacterial resistance to biocides in $\mathrm{h}$ health care environment: should it be of genuine concern? J Hosp Infect 65 (suppl 2), 60-72.

McDonnell G, and Russell AD (1999) Antiseptics and disinfectants: activity, action and resistance. Clin Microbiol Rev 12,147-179.

National Committee for Clinical Laboratory Standards (1993) Performance standards for antimicrobial disc susceptibility tests: approved standard. 4th ed. National Committee For Clinical Laboratory Standards Document M2-A4. Villanova, PA: National Committee For Clinical Laboratory Standards.

National Committee for Clinical Laboratory Standards (1997) Specialty Collection: Susceptibility Testing. SC21-L. M7A4. NCCLS, Wayne, PA.

Poole K (2007) Efflux pumps a antimicrobial resistance mechanisms. Ann Med 39, 162-76.

Russell AD (2003) Biocide use and antibiotic resistance: the relevance of laboratory findings to clinical and environmental situations. Lancet Infect Dis 3,794-803.

Russell AD, and McDonnell,G (2000) A major factor in studying biocidal action. J Hosp Infect 44, 1-3. 
Russell AD, Suller MTE, and Maillard JY (1999) Do antiseptics and disinfectants select for antibiotic resistance? J Med Microbiol 48, 613-615.

Russell AD, Tattawasart U, Maillard JY, and Furr JR (1998) Possible link between bacterial resistance and use of antibiotics and biocides. Antimicrob Agents Chemother 42, 2151-2151.

SCENIHR (Scientific Committee on Emerging and Newly Identified Health Risks), Assessment of the Antibiotic Resistance Effects of Biocides, 19 January 2009. http:ec.europa.eu/health/ph risk/risk en.htm

Sheldon AT (2005) Antiseptic "Resistance": Real or Perceived Threat? Clin Infect Dis 40, 1650-1656.
Suller MTE, and Russell AD. (1999) Antibiotic and biocide resistance in methicillin resistant Staphylococcus aureus and vancomycin- resistant Enterococcus. J Hosp Infect 43, 281291.

Walsh SE, Maillard JY, Russell AD, Charbonneau DL, Bartolo RG, and Catrenich C (2003) Development of bacterial resistance to several biocides and effects on antibiotic susceptibility. J Hosp Infect 55, 98-107.

Weber DJ, and Rutala WA (2006) Use of Germicides in the Home and the Healthcare Setting: Is There a Relationship between Germicide use and antibiotic resistance? Infect Control Hosp Epidemiol 27, 11071119.

\section{طريقة تحليل مقترحة لاستدخدام التركيزات الانيا المثبطة للنمو في المكورات العنقودية لايجاد علاقات

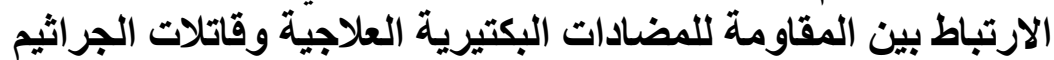

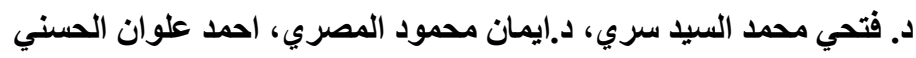 \\ قسم المبكروبيولوجيا والمناعة، كلية الصيدلة، جامعة الزقازيق}

استهدفت الدر اسة محاولة الربط بين نقص حساسية المكور ات العنقودية لقاتلات الجر اثيم و المقاومة للمضادات الميكروبية

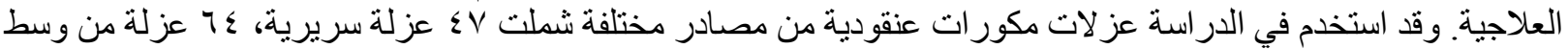

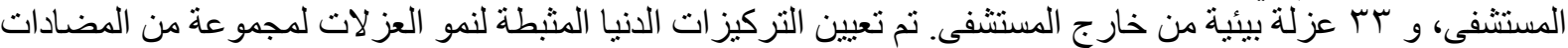

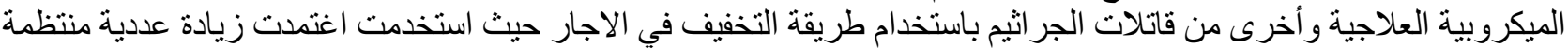

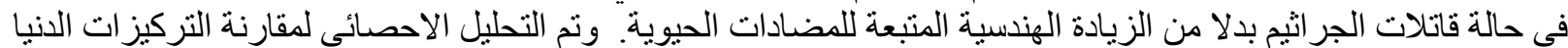

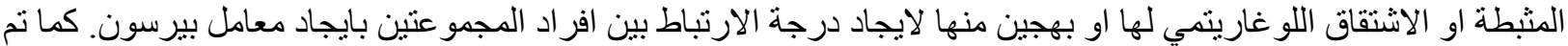

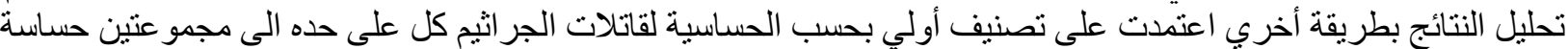

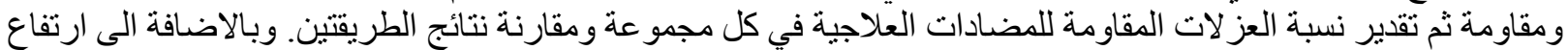

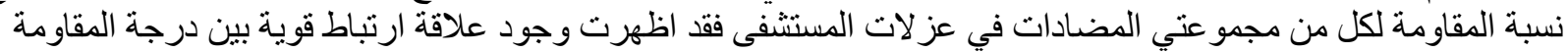

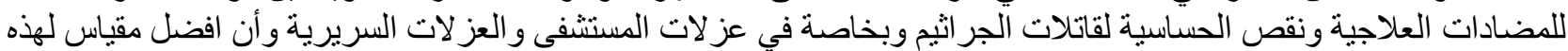

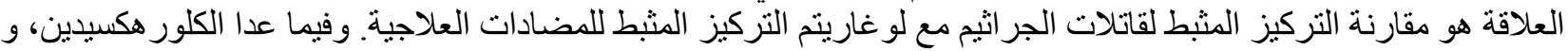

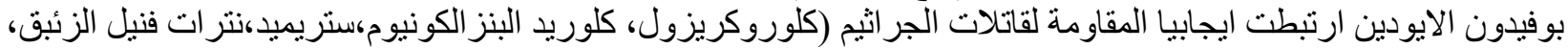
برومبد الايثيديم) بالمقاومة للمضادات العلاجية فيما عدا فانكو مايسين (وبعض الاستثناءات مع ثتر اسيكلين وسبرو فلوكساسين). فيما

بتعلق بالعز لات البيئية فقد كانت درجة المقاومة منخفضة نسبيا ووجدت علاقة ارتباط ايجابية بين المقاومة للمضادات العلاجية فئية وكل من كلوريد البنز الكونيوم وستريميد 